\title{
OP Opeys (Oncopathology \\ ROLE AND RELEVANCE OF THE EUROFLOW ANTIBODY PANEL IN IMMUNOPHENOTYPING OF CHRONIC LYMPHOPROLIFERATIVE DISORDERS
}

\begin{tabular}{ll}
\hline Dr Jyoti Sawhney* & Assistant Professor, Department of Oncopathology, GCRI. *Corresponding Author \\
\hline Dr Aparna Singh & Fellow Oncopathology, Department of Oncopathology, GCRI. \\
\hline Dr. Birwa Rahiya & Research Assistant, Flow cytometry division, GCRI. \\
\hline
\end{tabular}

ABSTRACT Detection of phenotypically aberrant and clonal mature lymphocytes is the diagnostic hallmark of chronic lymphoproliferative disorders (CLPD). B-CLPD is the commonest of all the CLPD. In this study we evaluated the role of CD200 and CD43 new markers that have been introduced in the Euroflow panel in the separation between chronic lymphocytic leukemia (CLL) and all other mature B cell malignancies. These markers were also correlated with the markers used in the Matutes score like FMC7 and Surface membrane Immunoglobulin. Patient samples between October 2017-February 2018, peripheral blood or bone marrow aspirates of patients with suspected B-cell lymphoproliferative disorders were subjected to evaluation by flow cytometry. After washing, samples were stained by antibodies targeting the antigens CD45, CD19, CD5, CD10, CD20, CD23, CD43, CD79b, CD200, FMC7, CD25,CD103 ,CD11c ,sIgM, kappa and lambda. Immunophenotyping was performed using a BD FACS Canto flow cytometer. There were a total of 108 cases of B CLPD that were analysed by flow cytometry. Mean age (SD) was 65 years. There were 68 males and 40 female patients. There were 71 cases of typical CLL , 7 cases of atypical CLL , 3 cases of Mantle cell lymphoma (MCL) , 5 cases of Follicular lymphoma ( FCL ), 8 cases of Splenic lymphoma with villous lymphocytes (SLVL), 6 cases of Hairy cell leukemia ( HCL) , 6 cases of unclassifiable B-NHL and 2 cases of lymphoplasmacytic lymphoma. All our cases of typical CLL showed bright expression of CD200 .It was also brightly expressed in all our atypical cases of CLL . CD43 was brightly positive in all our cases of typical CLL. The expression of this marker along with CD200 negativity was helpful in diagnosing of MCL. There were diagnostic difficulties in differentiating atypical CLL from B-NHL unclassifiable. The lack or dim expression FMC7 expression in all these cases along with absent or dim expression of sIgM were informative. Their use along with the panel by Euroflow is suggested to provide an accurate diagnosis.

\section{KEYWORDS : B chronic lymphoproliferative,Euroflow,CD200,CD43}

\section{INTRODUCTION}

Detection of phenotypically aberrant and clonal mature lymphocytes is the diagnostic hallmark of chronic lymphoproliferative disorders (CLPD).The reproducible diagnosis of CLPD based on morphology remains a challenge. The EuroFlow group has designed and evaluated an 8 color, 12 marker combination of antibodies for detection of phenotypically aberrant populations of mature $\mathrm{B}, \mathrm{T}$ and $\mathrm{NK}$ cells in peripheral blood ,bone marrow that is called lymphoid screening tube (LST)(1). B-CLPD is the commonest of all the CLPD. In this study we evaluated the role of CD200 and CD43 the new markers that have been introduced in the Euroflow panel in the separation between chronic lymphocytic leukemia (CLL) and other mature B cell malignancies. These markers were also assessed along with older markers used in the Matutes score like FMC7 and Surface membrane Immunoglobulin $(\operatorname{sIgM})(2,3)$.

\section{MATERIALS AND METHODS}

Patient samples between October 2017 to October 2018 were evaluated prospectively for diagnosis of B-CLPD . Peripheral blood or bone marrow aspirates were subjected to evaluation by flow cytometry. Immunophenotyping of peripheral blood or bone marrow aspirate was subjected to erythrocyte lysis by ammonium chloride. After washing, samples were stained by antibodies targeting the antigens CD45, CD19, CD5, CD10, CD20, CD23, CD43, CD79b, CD200, FMC7, CD25,CD103,CD11c sIgM, kappa and lambda. Corresponding isotype controls were used. Immunophenotyping was performed using a BD FACS Canto flow cytometer . Lymphocytes were selected by gating on CD45 high/sideways scatter (SSC)low events. All subsequent analyses were then performed on CD19+ B cells. Markers were considered positive when expression was $\geq 20 \%$ on the lymphoid cells. Mean Fluorescence Intensity (MFI) ratio (MFI sample/MFI isotype) was calculated as a measure of expression intensity.Diagnosis of MCL was confirmed by immunohistochemical detection of cyclin D1 in tissue biopsies or detection of $\mathrm{t}(11,14)(\mathrm{q} 13 ; 32)$ by FISH.

\section{STATISTICALANALYSIS}

Data analysis was performed using SPSS 15 for Windows (SPSS Inc., Chicago, IL, USA). Descriptive statistics were expressed as numbers and percentages. Categorical data were analyzed by multivariate forward stepwise regression analysis, Pearson's chi-square test, or Fisher's exact test as appropriate. A p-value of less than 0.05 was considered statistically significant

\section{RESULTS}

There were a total of 108 cases of B CLPD that were analysed by flow cytometry. Mean age was 65 years. There were 68 males and 40 female patients. There were 71 cases of typical CLL , 7 cases of atypical CLL , 3 cases of Mantle cell lymphoma (MCL), 5 cases of Follicular lymphoma ( FCL ), 8 cases of Splenic lymphoma with villous lymphocytes (SLVL), 6 cases of Hairy cell leukemia (HCL), 6 cases of unclassifiable B-NHL and 2 cases of lymphoplasmacytic lymphoma. All the cases of typical CLL had a Matutes score of 4 and 5. All the 7 cases of atypical CLL had a Matutes score of 3. Of these 7 cases of atypical CLL , 2 cases were CD5 negative , 2 were CD23 negative and 3 cases had bright expression of Cd79b.. All cases of HCL and variant form had classical morphology on peripheral blood or bone marrow biopsy. There were 5 cases of SLVL where morphology was that of small atypical lymphoid cells or lymphoplasmacytoid appearance and differentiating them from other CLPD relied on clinical details of moderate to massive splenomegaly clinically or on abdominal USG

\section{CD200 expression in CD 5 + lymphomas}

There were 71 cases of typical CLL .All these cases presented with a homogeneous bright expression of $\mathrm{Cd} 200$.

In the 7 cases of atypical CLL, CD200 was brightly expressed with an intensity as seen in cases of typical CLL . This bright expression was also seen in the 2 cases that were CD5 positive and CD23 negative and mimicked MCL. The 3 cases of MCL were negative for CD200 expression $(P$ value $<0.0001)$ ( CD 200 negative MCL; Graph 1)

All the 6 cases of B- NHL unclassifiable had bright expression of CD200 though only one case showed CD5 positivity.

\section{CD200 expression in CD 5-lymphomas}

4 cases of FCL had absent to dim expression of CD200 and only 1 case had bright expression of CD200.5 cases of B-NHL unclassifiable also had bright expression of CD200 $(P$ value $<0.05)$.

In the 2 cases of LPL there was variable expression in which one had dim and the other had bright expression.

\section{Lymphomas with hairy/villous projections}

The 4 cases of HCL were CD200 positive with the highest intensity of 
Volume - 10 | Issue - 9 | September - 2020 | PRINT ISSN No. 2249 - 555X | DOI : 10.36106/ijar

fluorescence .There were 2 cases of Hairy cell leukemia variant that were CD200 negative. ( CD 200 negative HCLv; Graph 2)
Of the 7 SMZL cases there was variable degree of CD200 expression with absent expression in 1 case, dim to moderate in 4 cases and bright in 2 cases $(P$ value $=0.020)$
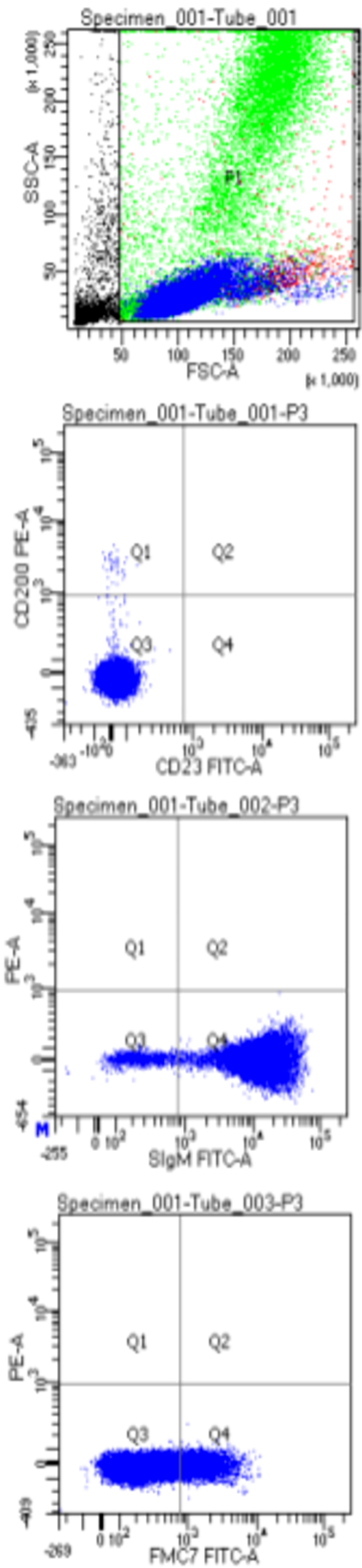
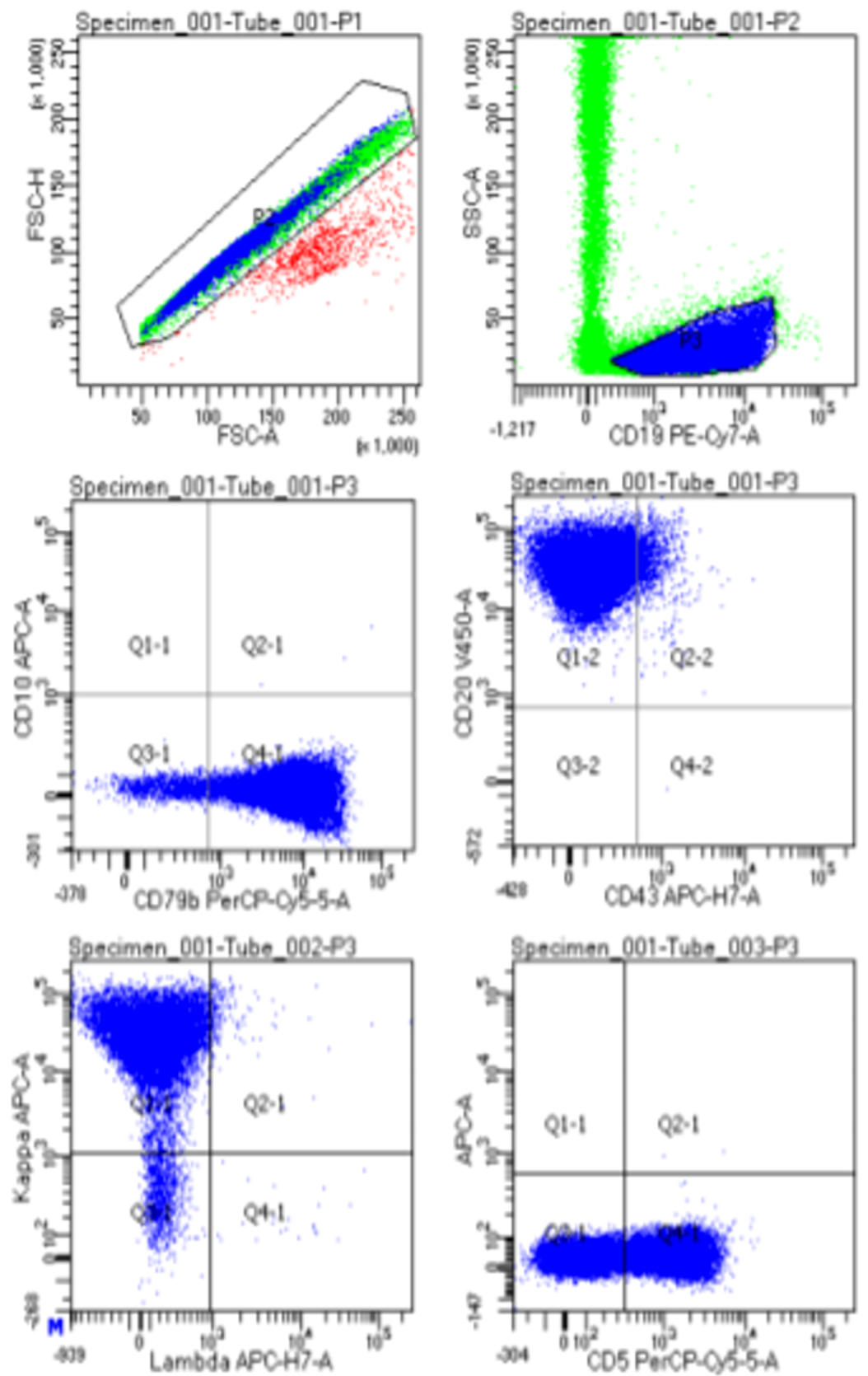
BD FACSDiva 8.0.2
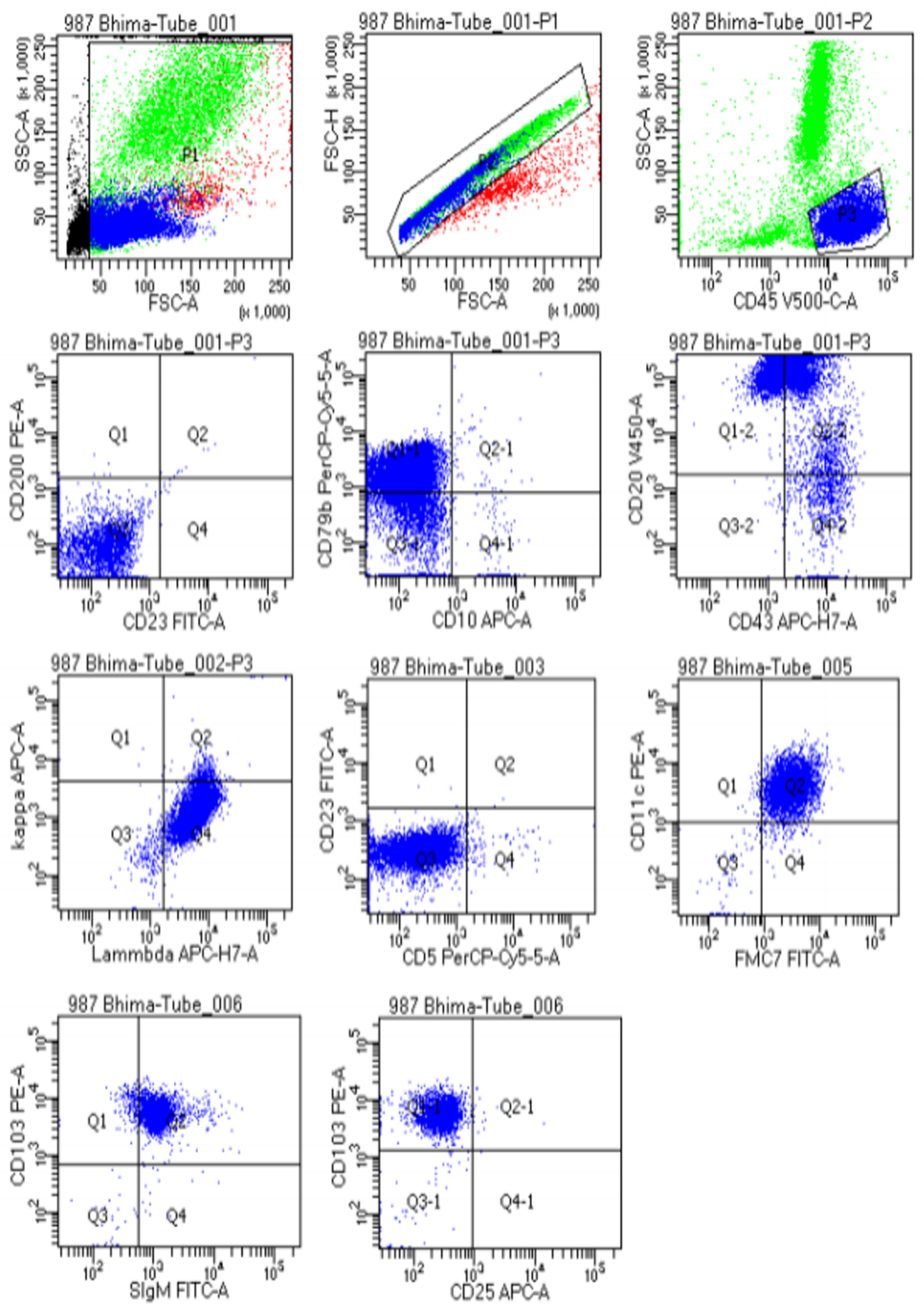

Graph 2 : CD 200 negative HCLv 


\section{Cd43 expression in CD5+ lymphomas}

In all 71 cases of typical CLL there was bright expression of CD43. All cases of atypical CLL had strong expression of Cd43.

In the 3 cases of MCL there was absent to dim expression of $\mathrm{Cd} 43$.

\section{CD43 expression in CD 5-lymphomas}

In 4 out of 5 cases of FCL, CD43 was negative while in 1 case there was strong expression of $\mathrm{Cd} 43$.

In 5 out of 6 B-NHL unclassifiable cases also CD43 was negative while it was strongly expressed in 1 case

In the 2 cases of LPL there was dim expression of Cd43.

\section{Lymphomas with hairy/villous projections}

In 6 cases of HCL,CD43 was negative in 1 case, dim in 2 cases and strong in 3 cases. In 8 cases of SLVL 2 cases were CD43 negative, 5 cases had dim to moderate expression and 1 case had bright expression of $\mathrm{Cd} 43$

Thus CD43 was bright positive in all CLL cases and also atypical CLL cases with Matutes score of 3 than non CLL cases ( $P$ value $<0.0001)$

Also when we compared relevance CD20 with that of FMC7 there was decreased sensitivity as well as specificity of in the differential diagnosis of CLL from non CLL cases compared to FMC7 ( sensitivity $68 \%$, specificity $62 \%$ )

\section{DISCUSSION}

In this study we investigated the expression of CD200 in all cases of mature B-CLPD and evaluated if the inclusion of this marker as suggested in the Euroflow panel would be useful to improve the diagnostic algorithm of B cell lymphomas by FCM. All our cases of typical CLL showed bright expression of CD200 .It was also brightly expressed in all our atypical cases of CLL . This was particularly relevant in all those atypical cases that were $\mathrm{CD} 5$ positive and $\mathrm{CD} 23$ negative with an expression pattern suggestive of MCL. The absence of CD200 in MCL helped in the differential diagnosis of the former from atypical CLL $(4,5)$. Thus the inclusion of CD200 was relevant in the primary panel as has been suggested by Euroflow. However there were two cases of CD200 negative B-CLPD that had the classical fried egg appearance on bone marrow biopsy with gross splenomegaly. On investigating these cases were CD103 positive with absence of CD25 expression with a diagnosis of Hairy cell leukemia variant.CD 200 negativity in Hairy cell leukemia variant has been reported by Matutes et al (6).All our cases of classical Hairy cell leukemia showed bright positivity for CD200. There was variable expression of CD 200 in cases diagnosed as Splenic lymphoma with villous lymphocytes.

In the category of $\mathrm{CD} 5$ negative lymphomas, the majority were follicular lymphoma and B-NHL unclassifiable. There was negative to dim expression of CD200 in all the FCL cases and bright expression of CD200 in all cases of B-NHL unclassifiable

CD43 is a surface molecule expressed on T cells , contrary to solid tissue, where CD43 is negative in normal B cells, in PB a normal subset (13-23\%) of B cells is CD43 positive (7).Euroflow has incorporated CD43 in differentiating various B cell lymphoproliferative disorders .CD43 was brightly positive in all our cases of typical CLL. There was negative to dim expression of CD43 in all our cases of Mantle cell lymphoma. The expression of this marker along with CD200 negativity was helpful in diagnosing of MCL(8). CD43 was particularly useful marker in differentiating cases of atypical CLL especially from cases of B-NHL unclassifiable.CD 43 was bright in atypical CLL in contrast to negative and dim expression of B-NHL unclassifiable. The consistently strong and bright expression of CD43 in all cases of typical CLL was the basis for evaluating two of our CD5 negative cases with dim expression of $\mathrm{CD} 23$ cases with other diagnostic markers like FMC7 and SIgM to differentiate these from BNHL unclassifiable. The negative expression of these markers indicated that these cases were that of atypical CLL

In our study despite using markers that have been suggested by the Euroflow consortium in the analysis of B chronic lymphoproliferative disorders we were faced with diagnostic difficulties in differentiating atypical CLL from B-NHL unclassifiable. It was in these cases that established markers especially the ones recommended in the Matutes score like FMC7 and SIgM were analysed further.The lack or dim expression FMC7 expression in all these cases along with absent or dim expression of sIgM were informative. FMC7 negativity proved to be a useful marker in the differential diagnosis of CLL as reported by some authors who have incorporated this marker in the CLL score ( $9,10,11)$.Our study supports the continued use of this marker in the secondary panel for such cases unlike reported by Euroflow that the marker is redundant and had no added value in the B-CLPD multitube antibody panel . It was suggested that FMC7 being an epitope of CD20 could be used interchangibly by CD20(12). However CD20 had markedly decreased sensitivity and specificity in differentiating CLL from non CLL cases and our analysis confirmed that CD20 was not useful in differentiating B-CLPD $(13,14)$. The staining patterns of sIgM were reproducible in all our cases B-CLPD unlike to reported by some authors (15). We support it' s use as a marker in the secondary panel for unclassifiable cases. The cases that were classified as B-NHL unclassifiable need further investigations on follow up like lymph node biopsy for accurate categorization of type of lymphoma.

Thus the present study underlines the significance of CD 200 as a valuable marker in the B-CLPD panel with relevance in diagnosing MCL.CD43 bright positivity was helpful in diagnosing typical and atypical CLL.However despite this we faced difficulties in differentiating some mature looking small cell B lymphomas like atypical CLL versus B-NHL unclassifiable.It was here that established markers like FMC7 and sIgM were helpful.Thus we support their use along with the panel suggested by Euroflow to provide a more accurate diagnosis.

\section{REFERENCES}

1) Van Dongen JJ ,Orfao A et al . Euroflow : Resetting leukemia and lymphoma immunophenotyping. Basis for companion diagnostics and personalized medicine.Leukemia 2012 Sep ;26(9): 1899-907

2) Matutes E, Owusu-Ankomah K, Morilla R, et al.The immunologicalprofile of B-cell disorders and proposal of a scoringsystem for the diagnosis of CLL Leukemia. 1994 Oct; 8(10):1640-5.

3) Moreau EJ, Matutes E, A'Hern RP, Morilla AM, Morilla RM et al.Improvement of the chronic lymphocytic leukemiascoringsystem with the monoclonal antibody SN8 (CD79b).Am J Clin Pathol. 1997 Oct;108(4):378-82

4) Sandes AF, de Lourdes Chauffaille $\mathrm{M}$ et al.CD200 has an important role in the differential diagnosis of mature B-cell neoplasms by multiparameter flow cytometry.. Cytometry B Clin Cytom. 2014 Mar;86(2):98-105.

5) Palumbo GA, Parrinello $\mathrm{N}$ et al .CD200 expression may help in differential diagnosis between mantle cell lymphoma and B-cell chronic lymphocytic leukemia. Leuk Res. 2009 Sep;33(9):1212-6

6) Matutes E, Martínez-Trillos A, et al.Hairy cell leukaemia-variant: Disease features and treatment.BestPract Res Clin Haematol. 2015 Dec;28(4):253-63

7) Sorigue $\mathrm{M}$, Juncà $\mathrm{J}$, et al. Expression of CD43 in chronic lymphoproliferative leukemias.Cytometry B Clin Cytom. 2018 Jan;94(1):136-142

8) Falay M, Afacan et al.The Role of CD200 and CD43 Expression in Differential Diagnosis between Chronic Lymphocytic Leukemia and Mantle Cell Lymphoma. Falay M, Afacan Öztürk B, Güneş K, Kalpakçı Y, Dağdaş S, Ceran F, Özet G.Turk J Haematol. 2018 May 25;35(2):94-98.

9) Köhnke T, Wittmann VK, Bücklein VL, et al.Diagnosis of CLL revisited: increased specificity by a modified five-marker scoring system including CD200.Köhnke T, specificity by a modified five-marker scoring system
Wittmann VK, Br J Haematol. 2017 Nov;179(3):480-487

10) Starostka D, Kriegova E, KudelkaM et alQuantitative assessment of informative immunophenotypic markers increases the diagnostic value of immunophenotyping in mature CD5-positive B-cell neoplasms.Cytometry B Clin Cytom. 2018 Jul;94(4):576587

11) Sorigue M, Franch-Sarto M, et aUsefulness of the CLLflow score. Cytometry B Clin Cytom. 2018 Jan 6. doi: 10.1002/cyto.b.21623

12) Deans, J.P. \&Polyak, M.J. (2008) FMC7 is an epitope of CD20. Blood, 111, 2492; author reply2493-2494.

13) Delgado, J., Matutes, E., Morilla, A.M., Morilla,R.M., Owusu-Ankomah, K.A,et al.(2003) Diagnostic significance of CD20 and FMC7 expression in B-cell disorders. AmericanJournal of Clinical Pathology, 120, 754-759

14) Poongodi RVarma $\mathrm{N}$ et al .Utility of CD200 expression and CD20 antibody binding capacity in differentiating chronic lymphocytic leukemia from other chronic lymphoproliferative disorders. Indian J Pathol Microbiol. 2018 Jan-Mar;61(1):50-57

15) Grier, D.D, Al-Quran, S.Z., Cardona, D.M., Li, Y.\&Braylan, R.C. (2012) Flow cytometric analysisof immunoglobulin heavy chain expression inB-cell lymphoma and reactive lymphoid hyperplasia. International Journal of Clinical and Experimental Pathology, 5, 110-118. 